\title{
THE ABSOLUTE CONTINUITY OF PHASE OPERATORS
}

\author{
BY
}

\section{J. DOMBROWSKI AND G. H. FRICKE}

ABSTRACT. This paper studies the spectral properties of a class of operators known as phase operators which originated in the study of harmonic oscillator phase. Ifantis conjectured that such operators had no point spectrum. It was later shown that certain phase operators were, in fact, absolutely continuous and that all phase operators at least had an absolutely continuous part. The present work completes the discussion by showing that all phase operators are absolutely continuous.

Introduction. Let $H$ be an infinite dimensional separable Hilbert space with orthonormal basis $\left\{\phi_{n}\right\}_{n=1}^{\infty}$. For any bounded linear operator $A$ on $H$, let $\operatorname{Sp}(A)$ denote the spectrum of $A$. If $A$ is selfadjoint with spectral resolution $A=$ $\int \lambda d E_{\lambda}$, denote by $H_{a}(A)$ the set of elements $x$ in $H$ for which $\left\|E_{\lambda} x\right\|^{2}$ is an absolutely continuous function of $\lambda$. It can be shown [2, p. 104] that $H_{a}(A)$ is a subspace of $H$ which reduces $A$. The restriction of $A$ to $H_{a}(A)$ is called the absolutely continuous part of $A$, and, if $H_{a}(A)=H$, the operator $A$ is said to be absolutely continuous.

Let $V$ denote the unilateral shift operator on $H$, so that $V \phi_{n}=\phi_{n+1}$. Furthermore, let $\left\{a_{n}\right\}_{n=1}^{\infty}$ be any sequence of positive real numbers converging monotonically to 1 and satisfying the following "chain sequence" condition:

$$
1 / 4 a_{n}^{2}=\left(1-g_{n-1}\right) g_{n}
$$

where $0 \leqslant g_{0}<1$ and $0<g_{n}<1 \quad(n>0)$.

Define the operator $A$ by $A \phi_{n}=a_{n-1} \phi_{n}$ with $a_{0}=0$. Consider now the following operators on $\mathrm{H}$ :

$$
C=\left(V^{*} A+A V\right) / 2, \quad S=\left(V^{*} A-A V\right) / 2 i .
$$

Such operators, called phase operators, have been studied in conjunction with the phase of the harmonic oscillator. Note that $C$ and $S$ are the real and imaginary parts, respectively, of $T=V^{*} A$ and, in particular, are selfadjoint. The conditions on the sequence $\left\{a_{n}\right\}_{n=1}^{\infty}$ guarantee that $\operatorname{Sp}(C)=\mathrm{Sp}(S)=[-1,1]$. [For a more detailed discussion of the origin of such operators and their properties see [3],

Presented to the Society, January 25, 1975; received by the editors November 15, 1974. AMS (MOS) subject classifications (1970). Primary 47B15, $47 \mathrm{~B} 47$.

Key words and phrases. Phase operators, absolute continuity of phase operators, real parts of weighted shifts. 
[4], [5] and the references cited there.] Since $C$ and $S$ are unitarily equivalent (see [3]), it is sufficient to consider the spectral properties of $C$.

It follows from results due to Putnam that those phase operators for which the corresponding sequence $\left\{a_{n}\right\}_{n=1}^{\infty}$ increases to 1 are absolutely continuous. (See [1].) Also, it was shown by one of the authors in a previous work [1] that those phase operators for which the corresponding sequence $\left\{a_{n}\right\}_{n=1}^{\infty}$ decreases monotonically to 1 have an absolutely continuous part whose spectrum is exactly the interval $[-1,1]$. It was not known at that time, however, if phase operators of this type were, in fact, absolutely continuous. It will be shown below that they are.

Preliminary results. The aim now is to show that every phase operator $C$, for which the corresponding sequence $\left\{a_{n}\right\}_{n=1}^{\infty}$ decreases monotonically to 1 , is absolutely continuous. The following results are needed.

LEMMA 1. Every element $\phi_{n}$ of the given orthonormal basis for $\mathrm{H}$ can be expressed in the form $P_{n}(C) \phi_{1}$ where $P_{n}(C)$ denotes a polynomial in $C$.

Proof. Clearly $P_{1}(C) \equiv I$. Since $C \phi_{1}=a_{1} \phi_{2} / 2$ it follows that $\phi_{2}=$ $2 C \phi_{1} / a_{1}$ and $P_{2}(C)=2 C / a_{1}$. Assume now that the lemma has been verified for $n=1,2, \ldots, k$ and consider $\phi_{k+1}$. Since

$$
C \phi_{k}=\left[a_{k-1} \phi_{k-1}+a_{k} \phi_{k+1}\right] / 2,
$$

it follows that

$$
\phi_{k+1}=\left[2 C \phi_{k}-a_{k-1} \phi_{k-1}\right] / a_{k} \text {, }
$$

and hence that

$$
P_{k+1}(C)=\left[2 C P_{k}(C)-a_{k-1} P_{k-1}(C)\right] / a_{k} .
$$

It will be shown below that $\phi_{1} \in H_{a}(C)$. By the previous lemma and the fact that the subspace $H_{a}(C)$ is invariant under $C$, it will follow immediately that $C$ is absolutely continuous. To this end let $C=\int \lambda d E_{\lambda}$ be the spectral decomposition of $C$, and let $E(\beta)$ denote the projection operator associated with any Borel set $\beta$ of the real line. It follows from the spectral theorem for selfadjoint operators that if $C=\int \lambda d E_{\lambda}$, then $P_{n}(C)=\int P_{n}(\lambda) d E_{\lambda}$. Thus the sequence of operators $\left\{P_{n}(C)\right\}_{n=1}^{\infty}$ defines a sequence of polynomials $\left\{P_{n}(\lambda)\right\}_{n=1}^{\infty}$ where

$$
P_{1}(\lambda) \equiv 1, \quad P_{2}(\lambda)=2 \lambda / a_{1},
$$

and for $n \geqslant 3$,

$$
P_{n}(\lambda)=\left[2 \lambda P_{n-1}(\lambda)-a_{n-2} P_{n-2}(\lambda)\right] / a_{n-1} .
$$

LEMMA 2. The polynomials $\left\{P_{n}(\lambda)\right\}_{n=1}^{\infty}$ are orthonormal with respect to the measure $\mu$ defined for every Borel set $\beta$ of the real line by $\mu(\beta)=\left\|E(\beta) \phi_{1}\right\|^{2}$. That is, 


$$
\int_{[-1,1]} P_{n}(\lambda) P_{m}(\lambda) d\left\|E_{\lambda} \phi_{1}\right\|^{2}= \begin{cases}1, & n=m, \\ 0, & n \neq m .\end{cases}
$$

(The support of the measure is the interval $[-1,1]$ since $\operatorname{Sp}(C)=[-1,1]$.)

Proof. It is enough to note that

$$
\int_{[-1,1]} P_{n}(\lambda) P_{m}(\lambda) d\left\|E_{\lambda} \phi_{1}\right\|^{2}=\left\langle P_{m}(C) \phi_{1}, P_{n}(C) \phi_{1}\right\rangle=\left\langle\phi_{m}, \phi_{n}\right\rangle .
$$

Recall now that the sequence $\left\{a_{n}\right\}_{n=1}^{\infty}$ corresponding to the phase operator $C$ satisfies the "chain sequence" condition (1). The related sequence $\left\{g_{n}\right\}_{n=0}^{\infty}$ is not unique unless it is assumed that $g_{0}=0$. (That it is always possible to choose $g_{0}=0$ is clear from the proof of the "chain sequence" condition as presented in [5].)

LEMMA 3. If $g_{0}=0$, then for $n=2,3,4, \ldots, 2 g_{n-1} P_{n}(1)=a_{n-1} P_{n-1}$ (1).

Proof. If $g_{0}=0$ then $a_{1}^{2}=4 g_{1}$. Since $P_{1}(\lambda) \equiv 1$ and $P_{2}(\lambda)=2 \lambda / a_{1}$, it follows that

$$
2 g_{1} P_{2}(1)=2 \cdot a_{1}^{2} / 4 \cdot 2 / a_{1}=a_{1}=a_{1} P_{1}(1),
$$

and the lemma is true for $n=2$. Assume now that the lemma has been verified for $n=2, \ldots, k$ and consider $n=k+1$. From (2) and the fact that $2 g_{k-1} P_{k}(1)=a_{k-1} P_{k-1}(1)$, it follows that

$$
\begin{aligned}
2 g_{k} P_{k+1}(1) & =\left(2 g_{k} / a_{k}\right)\left[2 P_{k}(1)-a_{k-1} P_{k-1}(1)\right] \\
& =\left(2 g_{k} / a_{k}\right)\left[2 P_{k}(1)-2 g_{k-1} P_{k}(1)\right] \\
& =\left(4 g_{k} / a_{k}\right)\left(1-g_{k-1}\right) P_{k}(1)=a_{k} P_{k}(1),
\end{aligned}
$$

as was to be shown.

It was shown in [1] that if the sequence $\left\{a_{n}\right\}_{n=1}^{\infty}$ monotonically decreases to 1 , the related sequence $\left\{g_{n}\right\}_{n=0}^{\infty}$ (with $g_{0}=0$ ) monotonically increases to $1 / 2$. This result will be used in

LemMa 4. For $n=1,2,3, \ldots, P_{n}(1) \geqslant 1$.

Proof. Note first that $a_{1}^{2}=4 g_{1}$ and $g_{1} \leqslant 1 / 2$ imply that $a_{1}^{2} \leqslant 2$. Since $P_{1}(\lambda) \equiv 1$ and $P_{2}(\lambda)=2 \lambda / a_{1}$, the result is clear for $n=1,2$. Now assume it has been shown for $n=1,2, \ldots, k$. The recursion formula (2) and the previous lemma imply that

$$
\begin{aligned}
P_{k+1}(1) & =\left[2 P_{k}(1)-a_{k-1} P_{k-1}(1)\right] / a_{k} \\
& =\left[\left(a_{k-1} / g_{k-1}\right) P_{k-1}(1)-a_{k-1} P_{k-1}(1)\right] / a_{k} \\
& \geqslant\left(a_{k-1} / a_{k}\right) P_{k-1}(1) \quad\left(\text { since } g_{k-1} \leqslant 1 / 2\right) \\
& \geqslant 1
\end{aligned}
$$


Lemma 5. For $\lambda \in[-1,1]$ define

$$
S_{N}(\lambda)=\sum_{n=1}^{N} \frac{\left(a_{n}^{2}-a_{n+1}^{2}\right)}{a_{1}^{2}} P_{n+1}^{2}(\lambda) ; \quad N=1,2, \ldots
$$

Then

$$
\begin{aligned}
1-S_{N}(\lambda)= & \left(1 / a_{1}^{2}\right)\left[a_{N}^{2} P_{N}^{2}(\lambda)-2 \lambda a_{N} P_{N}(\lambda) P_{N+1}(\lambda)+P_{N+1}^{2}(\lambda)\right] \\
& +\left(\left(a_{N+1}^{2}-1\right) / a_{1}^{2}\right) P_{N+1}^{2}(\lambda)
\end{aligned}
$$

so that, in particular,

$$
S_{N}(\lambda) \leqslant 1
$$

Proof. Suppose $N=1$. Then

$$
\begin{aligned}
1-S_{1}(\lambda) & =1-\frac{\left(a_{1}^{2}-a_{2}^{2}\right)}{a_{1}^{2}} P_{2}^{2}(\lambda) \\
& =1-\frac{\left(a_{1}^{2}-1\right)}{a_{1}^{2}} \frac{4 \lambda^{2}}{a_{1}^{2}}+\frac{\left(a_{2}^{2}-1\right)}{a_{1}^{2}} P_{2}^{2}(\lambda) \\
& =\frac{1}{a_{1}^{2}}\left[a_{1}^{2} P_{1}^{2}(\lambda)-2 \lambda a_{1} P_{1}(\lambda) P_{2}(\lambda)+P_{2}^{2}(\lambda)\right]+\frac{\left(a_{2}^{2}-1\right)}{a_{1}^{2}} P_{2}^{2}(\lambda) .
\end{aligned}
$$

Hence (3) is true for $N=1$. Assume now that (3) has been verified for $N=1$, $\ldots, k$, and consider

$$
S_{k+1}(\lambda)=S_{k}(\lambda)+\frac{\left(a_{k+1}^{2}-a_{k+2}^{2}\right)}{a_{1}^{2}} P_{k+2}^{2}(\lambda)
$$

Then

$$
\begin{aligned}
1-S_{k+1}(\lambda)= & 1-S_{k}(\lambda)-\frac{\left(a_{k+1}^{2}-1\right)}{a_{1}^{2}} P_{k+2}^{2}(\lambda) \\
& +\frac{\left(a_{k+2}^{2}-1\right)}{a_{1}^{2}} P_{k+2}^{2}(\lambda) .
\end{aligned}
$$

Since

$$
1-S_{k}(\lambda)=\frac{1}{a_{1}^{2}}\left[a_{k}^{2} P_{k}^{2}(\lambda)-2 \lambda a_{k} P_{k}(\lambda) P_{k+1}(\lambda)+a_{k+1}^{2} P_{k+1}^{2}(\lambda)\right]
$$

and

$$
a_{k+1}^{2} P_{k+2}^{2}(\lambda)=\left[2 \lambda P_{k+1}(\lambda)-a_{k} P_{k}(\lambda)\right]^{2}
$$

it follows that 


$$
\begin{aligned}
1-S_{k+1}(\lambda)= & \frac{1}{a_{1}^{2}}\left[a_{k+1}^{2} P_{k+1}^{2}(\lambda)+2 \lambda a_{k} P_{k}(\lambda) P_{k+1}(\lambda)-4 \lambda^{2} P_{k+1}^{2}(\lambda)+P_{k+2}^{2}(\lambda)\right] \\
& +\left(\left(a_{k+2}^{2}-1\right) / a_{1}^{2}\right) P_{k+2}^{2}(\lambda) \\
= & \frac{1}{a_{1}^{2}}\left\{a_{k+1}^{2} P_{k+1}^{2}(\lambda)-2 \lambda a_{k+1} P_{k+1}(\lambda)\right. \\
& \left.\quad \cdot\left[\frac{2 \lambda P_{k+1}(\lambda)-a_{k} P_{k}(\lambda)}{a_{k+1}}\right]+P_{k+2}^{2}(\lambda)\right\} \\
& +\left(\left(a_{k+2}^{2}-1\right) / a_{1}^{2}\right) P_{k+2}^{2}(\lambda) \\
= & \frac{1}{a_{1}^{2}}\left[a_{k+1}^{2} P_{k+1}^{2}(\lambda)-2 \lambda a_{k+1} P_{k+1}(\lambda) P_{k+2}(\lambda)+P_{k+2}^{2}(\lambda)\right] \\
& +\left(\left(a_{k+2}^{2}-1\right) / a_{1}^{2}\right) P_{k+2}^{2}(\lambda)
\end{aligned}
$$

and (3) is verified for $N=k+1$.

Finally, (4) follows from (3) and the observation that

$$
\left|2 \lambda a_{N} P_{N}(\lambda) P_{N+1}(\lambda)\right| \leqslant 2 a_{N}\left|P_{N}(\lambda) P_{N+1}(\lambda)\right| \leqslant a_{N}^{2} P_{N}^{2}(\lambda)+P_{N+1}^{2}(\lambda) .
$$

LEMma 6. For $k>1$ and any interval $\Delta$ such that $\Delta \subset(-1+1 / k, 1-$ $1 / k)$,

(5) $\int_{\Delta} S_{N}(\lambda) d\left\|E_{\lambda} \phi_{1}\right\|^{2} \leqslant\left(1-\frac{1}{10 k}\right)\left\|E(\Delta) \phi_{1}\right\|^{2}, \quad N=1,2, \ldots$

Proof. Let $D_{N}(\lambda)=\left(\left|a_{N} P_{N}(\lambda)\right|-\left|P_{N+1}(\lambda)\right|\right)^{2}$. If $\left|P_{N}(\lambda)\right|>$ $3\left|P_{N+1}(\lambda)\right|$ then

$$
D_{N}(\lambda) \geqslant\left(3 a_{N}-1\right)^{2} P_{N+1}^{2}(\lambda) \geqslant 4 P_{N+1}^{2}(\lambda)
$$

and

$$
D_{N}(\lambda) \geqslant\left(a_{N}-1 / 3\right)^{2} P_{N}^{2}(\lambda) \geqslant(4 / 9) P_{N}^{2}(\lambda) .
$$

If $\left|P_{N}(\lambda)\right|<\left|P_{N+1}(\lambda)\right| / 3$, then

$$
D_{N}(\lambda) \geqslant\left(1-a_{N} / 3\right)^{2} P_{N+1}^{2}(\lambda) \geqslant(2 / 9) P_{N+1}^{2}(\lambda)
$$

and

$$
D_{N}(\lambda) \geqslant\left(3-a_{N}\right)^{2} P_{N}^{2}(\lambda) \geqslant 2 P_{N}^{2}(\lambda) .
$$

Thus $D_{N}(\lambda) \geqslant \max \left\{P_{N}^{2}(\lambda), P_{N+1}^{2}(\lambda)\right\} / 5$ whenever $\left|P_{N}(\lambda)\right| \notin\left[\left|P_{N+1}(\lambda)\right| / 3\right.$, $\left.3\left|P_{N+1}(\lambda)\right|\right]$. If $\left|P_{N}(\lambda)\right| \in\left[\left|P_{N+1}(\lambda)\right| / 3,3\left|P_{N+1}(\lambda)\right|\right]$, then 


$$
2 a_{N}\left|P_{N}(\lambda) P_{N+1}(\lambda)\right| / a_{1}^{2} \geqslant \max \left\{P_{N}^{2}(\lambda), P_{N+1}^{2}(\lambda)\right\} / 10 .
$$

Hence for any $\lambda \in(-1,1)$,

$$
\begin{aligned}
\left(1 / a_{1}^{2}\right)\left[a_{N}^{2} P_{N}^{2}(\lambda)-2 \lambda a_{N} P_{N}(\lambda) P_{N+1}+P_{N+1}^{2}(\lambda)\right] \\
\quad \geqslant\left(1 / a_{1}^{2}\right)\left[\left(a_{N}\left|P_{N}(\lambda)\right|-\left|P_{N+1}(\lambda)\right|\right)^{2}+2(1-|\lambda|) a_{N}\left|P_{N}(\lambda) P_{N+1}(\lambda)\right|\right] \\
\quad \geqslant(1 / 10)(1-|\lambda|) \max \left\{P_{N}^{2}(\lambda), P_{N+1}^{2}(\lambda)\right\} .
\end{aligned}
$$

Now consider an interval $\Delta$ such that $\Delta \subset(-1+1 / k, 1-1 / k)$. To verify (5) it is necessary to consider several cases.

Case I. Suppose $\int_{\Delta} P_{n}^{2}(\lambda) d\left\|E_{\lambda} \phi_{1}\right\|^{2} \leqslant\left\|E(\Delta) \phi_{1}\right\|^{2}$ for all $n$. Then

$$
\begin{aligned}
\int_{\Delta} S_{N}(\lambda) d\left\|E_{\lambda} \phi_{1}\right\|^{2} & =\sum_{n=1}^{N} \frac{a_{n}^{2}-a_{n+1}^{2}}{a_{1}^{2}} \int_{\Delta} P_{n+1}^{2}(\lambda) d\left\|E_{\lambda} \phi_{1}\right\|^{2} \\
& \leqslant \sum_{n=1}^{N} \frac{a_{n}^{2}-a_{n+1}^{2}}{a_{1}^{2}}\left\|E(\Delta) \phi_{1}\right\|^{2} \\
& \leqslant\left(1-1 / a_{1}^{2}\right)\left\|E(\Delta) \phi_{1}\right\|^{2} \leqslant 1 / 2\left\|E(\Delta) \phi_{1}\right\|^{2} .
\end{aligned}
$$

Case II. Suppose there exists a subsequence $n_{i}, n_{i} \rightarrow \infty$, for which $\int_{\Delta} P_{n_{i}}^{2}(\lambda) d\left\|E_{\lambda} \phi_{1}\right\|^{2}>\left\|E(\Delta) \phi_{1}\right\|^{2}$. If $\int_{\Delta} P_{N}^{2}(\lambda) d\left\|E_{\lambda} \phi_{1}\right\|^{2}>\left\|E(\Delta) \phi_{1}\right\|^{2}$, it follows from (6) that

$$
\int_{\Delta}\left(1-S_{N}(\lambda)\right) d\left\|E_{\lambda} \phi_{1}\right\|^{2} \geqslant \int_{\Delta} \frac{1}{10}(1-|\lambda|) P_{N}^{2}(\lambda) d\left\|E_{\lambda} \phi_{1}\right\|^{2} \geqslant \frac{1}{10 k}\left\|E(\Delta) \phi_{1}\right\|^{2},
$$

and hence that

$$
\int_{\Delta} S_{N}(\lambda) d\left\|E_{\lambda} \phi_{1}\right\|^{2} \leqslant\left(1-\frac{1}{10 k}\right)\left\|E(\Delta) \phi_{1}\right\|^{2} .
$$

Furthermore, for fixed $\lambda, S_{n}(\lambda)$ increases with $n$ and hence

$$
\int_{\Delta} S_{n}(\lambda) d\left\|E_{\lambda} \phi_{1}\right\|^{2} \leqslant\left(1-\frac{1}{10 k}\right)\left\|E(\Delta) \phi_{1}\right\|^{2} \quad \text { for all } n \leqslant N .
$$

The argument is concluded by noting that $\int_{\Delta} P_{N}^{2}(\lambda) d\left\|E_{\lambda} \phi_{1}\right\|^{2}>\left\|E(\Delta) \phi_{1}\right\|^{2}$ for infinitely many values of $N$.

Case III. Suppose $\int_{\Delta} P_{n}^{2}(\lambda) d\left\|E_{\lambda} \phi_{1}\right\|^{2}>\left\|E(\Delta) \phi_{1}\right\|^{2}$ for finitely many values of $n$. Let $n_{0}$ be such that

$$
P=\int_{\Delta} P_{n_{0}}^{2}(\lambda) d\left\|E_{\lambda} \phi_{1}\right\|^{2}
$$

$$
\geqslant \int_{\Delta} P_{n}^{2}(\lambda) d\left\|E_{\lambda} \phi_{1}\right\|^{2} \quad \text { for all } n \text {. }
$$

Then for $n>n_{0}$, 


$$
\begin{aligned}
\int[1 & \left.-S_{n}(\lambda)\right] d\left\|E_{\lambda} \phi_{1}\right\|^{2} \\
& =\int_{\Delta}\left[1-S_{n_{0}-1}(\lambda)-\sum_{j=n_{0}}^{n} \frac{a_{j}^{2}-a_{j+1}^{2}}{a_{1}^{2}} P_{j+1}^{2}(\lambda)\right] d\left\|E_{\lambda} \phi_{1}\right\|^{2} \\
& =\int_{\Delta}\left[1-S_{n_{0}-1}(\lambda)\right] d\left\|E_{\lambda} \phi_{1}\right\|^{2}-\sum_{j=n_{0}}^{n} \frac{a_{j}^{2}-a_{j+1}^{2}}{a_{1}^{2}} \int_{\Delta} P_{j+1}^{2}(\lambda) d\left\|E_{\lambda} \phi_{1}\right\|^{2} .
\end{aligned}
$$

By (3), (6), and (7), it then follows that

$$
\begin{aligned}
\int_{\Delta}\left[1-S_{n}(\lambda)\right] d\left\|E_{\lambda} \phi_{1}\right\|^{2} \geqslant & \int_{\Delta} \frac{1}{10}(1-|\lambda|) P_{n_{0}}^{2}(\lambda) d\left\|E_{\lambda} \phi_{1}\right\|^{2} \\
& +\frac{a_{n_{0}}^{2}-1}{a_{1}^{2}} P-\sum_{j=n_{0}}^{n} \frac{a_{j}^{2}-a_{j+1}^{2}}{a_{1}^{2}} P \\
\geqslant & P / 10 k \geqslant\left\|E(\Delta) \phi_{1}\right\|^{2} / 10 k .
\end{aligned}
$$

Hence $\int_{\Delta} S_{n}(\lambda) d\left\|E_{\lambda} \phi_{1}\right\|^{2} \leqslant(1-1 / 10 k)\left\|E(\Delta) \phi_{1}\right\|^{2}$ for all $n$ as was to be shown.

LEMMA 7. $\int_{\{-1,1\}} d\left\|E_{\lambda} \phi_{1}\right\|^{2}=0$.

Proof. Recall that

$$
\int_{[-1,1]} P_{n}(\lambda) P_{m}(\lambda) d\left\|E_{\lambda} \phi_{1}\right\|^{2}= \begin{cases}1, & n=m, \\ 0, & n \neq m,\end{cases}
$$

and note that $P_{2 n}(\lambda)$, for $n=1,2, \ldots$, is a polynomial of degree $2 n-1$, symmetric with respect to the origin. Note also that $\int_{[-1,1]} \lambda^{2 n-1} P_{2 n+2}(\lambda) d\left\|E_{\lambda} \phi_{1}\right\|^{2}$ $=0$. For $L>0$ define $M_{n}=\left\{\lambda \in(-1,1)|| P_{2 n+2}(\lambda) \mid>L\right\}$. By Lemma 4, $P_{2 n+2}(1) \geqslant 1$. Hence

$$
\begin{aligned}
0= & \left|\int_{[-1,1]} \lambda^{2 n-1} P_{2 n+2}(\lambda) d\left\|E_{\lambda} \phi_{1}\right\|^{2}\right| \\
\geqslant & P_{2 n+2}(1) \int_{\{-1,1\}} d\left\|E_{\lambda} \phi_{1}\right\|^{2}-\int_{(-1,1)}\left|\lambda^{2 n-1}\right|\left|P_{2 n+2}(\lambda)\right| d\left\|E_{\lambda} \phi_{1}\right\|^{2} \\
\geqslant & \int_{\{-1,1\}} d\left\|E_{\lambda} \phi_{1}\right\|^{2}-\int_{M_{n}}\left|P_{2 n+2}(\lambda)\right| d\left\|E_{\lambda} \phi_{1}\right\|^{2} \\
& -\int_{(-1,1) / M_{n}}|\lambda|^{2 n-1} d\left\|E_{\lambda} \phi_{1}\right\|^{2} \\
\geqslant & \int_{\{-1,1\}} d\left\|E_{\lambda} \phi_{1}\right\|^{2}-\frac{1}{L} \int_{M_{n}} P_{2 n+2}^{2}(\lambda) d\left\|E_{\lambda} \phi_{1}\right\|^{2}-L \int_{(-1,1)}|\lambda|^{2 n-1} d\left\|E_{\lambda} \phi_{1}\right\|^{2} \\
\geqslant & \int_{\{-1,1\}} d\left\|E_{\lambda} \phi_{1}\right\|^{2}-\frac{1}{L}-L \int_{(-1,1)}|\lambda|^{2 n-1} d\left\|E_{\lambda} \phi_{1}\right\|^{2} .
\end{aligned}
$$


Since $n$ can be chosen arbitrarily large, it follows that $\int_{\{-1,1\}} d\left\|E_{\lambda} \phi_{1}\right\|^{2} \leqslant 1 / L$, and, since $L$ was arbitrary, that $\int_{\{-1,1\}} d\left\|E_{\lambda} \phi_{1}\right\|^{2}=0$.

It is easily verified that $C S-S C=-i K / 2$ where $K \phi_{1}=-a_{1}^{2} \phi_{1}$, and for $n>1, K \phi_{n}=\left(a_{n-1}^{2}-a_{n}^{2}\right) \phi_{n}$. The following result is adapted from a proof due to Putnam [6, p. 20].

LEMMA 8. For any finite interval $\Delta$,

$$
\left.K K E(\Delta) \phi_{1}, E(\Delta) \phi_{1}\right\rangle|\leqslant 2| \Delta \mid\left\|E(\Delta) \phi_{1}\right\|^{2} \text {. }
$$

ProOF. Let $\lambda_{0}$ be the midpoint of $\Delta$. It follows from the commutator equation $C S-S C=-i K / 2$ that

$$
E(\Delta)\left(C-\lambda_{0} I\right) S E(\Delta)-E(\Delta) S\left(C-\lambda_{0} I\right) E(\Delta)=(i / 2) E(\Delta) K E(\Delta),
$$

and hence that

$$
\begin{gathered}
\left\langle S E(\Delta) \phi_{1},\left(C-\lambda_{0} I\right) E(\Delta) \phi_{1}\right\rangle-\left\langle\left(C-\lambda_{0} I\right) E(\Delta) \phi_{1}, S E(\Delta) \phi_{1}\right\rangle \\
=-(i / 2)\left\langle K E(\Delta) \phi_{1}, E(\Delta) \phi_{1}\right\rangle .
\end{gathered}
$$

Then by the Schwarz inequality,

$$
1 / 2 \mid\left\langle K E(\Delta) \phi_{1}, E(\Delta) \phi_{1}\right\rangle \leqslant 2\left\|S E(\Delta) \phi_{1}\right\|\left\|\left(C-\lambda_{0} I\right) E(\Delta) \phi_{1}\right\| .
$$

Finally,

$$
\|\left(C-\lambda_{0} I E(\Delta) \phi_{1}\left\|^{2}=\int_{\Delta}\left|\lambda-\lambda_{0}\right|^{2} d\right\| E_{\lambda} \phi_{1} \|^{2}\right.
$$

implies that

$$
\|\left(C-\lambda_{0} \cap E(\Delta) \phi_{1}\|\leqslant 1 / 2|\Delta|\| E(\Delta) \phi_{1} \|\right.
$$

and, since $\|s\|=1$, that

$$
\left|K K E(\Delta) \phi_{1}, E(\Delta) \phi_{1}\right\rangle|\leqslant 2| \Delta \mid\left\|E(\Delta) \phi_{1}\right\|^{2}
$$

as was to be shown.

The main result. It remains now to combine the results of the previous section to obtain the main result of this paper.

THEOREM. Every phase operator $C$ for which the corresponding sequence $\left\{a_{n}\right\}_{n=1}^{\infty}$ decreases monotonically to 1 is absolutely continuous.

Proof. As noted above it is enough to show that $\phi_{1} \in H_{a}(C)$. For any interval $\Delta$,

$$
\begin{gathered}
E(\Delta) \phi_{1}=\sum_{n=1}^{\infty}\left\langle E(\Delta) \phi_{1}, \phi_{n}\right\rangle \phi_{n}, \\
K E(\Delta) \phi_{1}=-a_{1}^{2}\left\langle E(\Delta) \phi_{1}, \phi_{1}\right\rangle \phi_{1} \\
+\sum_{n=1}^{\infty}\left(a_{n}^{2}-a_{n+1}^{2}\right)\left\langle E(\Delta) \phi_{1}, \phi_{n+1}\right\rangle \phi_{n+1},
\end{gathered}
$$


and

$$
\begin{aligned}
\left\langle K E(\Delta) \phi_{1}, E(\Delta) \phi_{1}\right\rangle= & -a_{1}^{2}\left|E(\Delta) \phi_{1}\right|^{4} \\
& +\sum_{n=1}^{\infty}\left(a_{n}^{2}-a_{n+1}^{2}\right)\left|\left\langle E(\Delta) \phi_{1} \phi_{n+1}\right\rangle\right|^{2}
\end{aligned}
$$

It then follows from Lemma 8 that

(8) $\left|\left\|E(\Delta) \phi_{1}\right\|^{4}-\sum_{n=1}^{\infty} \frac{\left(a_{n}^{2}-a_{n+1}^{2}\right)}{a_{1}^{2}} K E(\Delta) \phi_{1}, \phi_{n+1}\right\rangle^{2}\left|\leqslant \frac{2}{a_{1}^{2}}\right| \Delta \mid\left\|E(\Delta) \phi_{1}\right\|^{2}$.

Furthermore, if $\Delta \subset(-1+1 / k, 1-1 / k)$, the following argument holds. Let

$$
M_{N}=\sum_{n=1}^{N} \frac{\left(a_{n}^{2}-a_{n+1}^{2}\right)}{a_{1}^{2}}\left|\left\langle E(\Delta) \phi_{1}, \phi_{n+1}\right\rangle\right|^{2}
$$

Then

$$
\begin{aligned}
M_{N} & =\sum_{n=1}^{N} \frac{\left(a_{n}^{2}-a_{n+1}^{2}\right)}{a_{1}^{2}} \mid\left\langle E(\Delta) \phi_{1}, P_{n+1}(C) \phi_{1}\right\rangle^{2} \quad \text { (Lemma 1) } \\
& =\sum_{n=1}^{N} \frac{\left(a_{n}^{2}-a_{n+1}^{2}\right)}{a_{1}^{2}}\left|\int_{\Delta} P_{n+1}(\lambda) d\left\|E_{\lambda} \phi_{1}\right\|^{2}\right|^{2} \quad \text { (spectral theorem) } \\
& \leqslant\left\|E(\Delta) \phi_{1}\right\|^{2} \sum_{n=1}^{N} \frac{\left(a_{n}^{2}-a_{n+1}^{2}\right)}{a_{1}^{2}} \int_{\Delta} P_{n+1}^{2}(\lambda) d\left\|E_{\lambda} \phi_{1}\right\|^{2} \\
& \leqslant\left\|E(\Delta) \phi_{1}\right\|^{2} \int_{\Delta} S_{N}(\lambda) d\left\|E_{\lambda} \phi_{1}\right\|^{2} \\
& \leqslant(1-1 / 10 k)\left\|E(\Delta) \phi_{1}\right\|^{4} \quad \text { (Lemma 6). }
\end{aligned}
$$

Hence, it follows from (8) that

$$
\begin{aligned}
\frac{1}{10 k}\left\|E(\Delta) \phi_{1}\right\|^{4} & \leqslant\left.\left|\left\|E(\Delta) \phi_{1}\right\|^{4}-\sum_{n=1}^{\infty} \frac{\left(a_{n}^{2}-a_{n+1}^{2}\right)}{a_{1}^{2}} K E(\Delta) \phi_{1}, \phi_{n+1}\right\rangle\right|^{2} \mid \\
& \leqslant 2|\Delta|\left\|E(\Delta) \phi_{1}\right\|^{2} / a_{1}
\end{aligned}
$$

or, equivalently, that

$$
\left\|E(\Delta) \phi_{1}\right\|^{2} \leqslant(10 k) 2|\Delta| / a_{1}^{2} .
$$

Consider first a Borel set $\beta$ such that $\beta \subset(-1+1 / k, 1-1 / k)$. Let $\left\{\Delta_{1}, \Delta_{2}, \ldots\right\}$ be a countable covering of $\beta$ by disjoint intervals such that $\Delta_{j} \subset$ $(-1+1 / k, 1-1 / k)$ for all $j$. Then 


$$
\left\|E(\beta) \phi_{1}\right\|^{2}=\sum_{j}\left\|E\left(\beta \cap \Delta_{j}\right) \phi_{1}\right\|^{2} \leqslant \sum_{j}\left\|E\left(\Delta_{j}\right) \phi_{1}\right\|^{2} \leqslant(10 k) \frac{2}{a_{1}^{2}} \sum_{j}\left|\Delta_{j}\right| .
$$

Since the argument holds for any such countable covering of $\beta$, it follows that

$$
\left\|E(\beta) \phi_{1}\right\|^{2} \leqslant 20 k(\text { meas } \beta) / a_{1}^{2},
$$

where "meas $\beta$ " denotes the Lebesgue measure of $\beta$. Thus if meas $\beta=0$, then $\left\|E(\beta) \phi_{1}\right\|^{2}=0$. Consider now an arbitrary Borel set $\beta$. By Lemma $7,\left\|E(\beta) \phi_{1}\right\|^{2}$ $=\left\|E(\beta \cap(-1,1)) \phi_{1}\right\|^{2}$. Furthermore, since $\beta \cap(-1,1)=\bigcup_{k} \beta_{k}$ where $\beta_{k} \subset$ $(-1+1 / k, 1-1 / k)$, it follows that

$$
\left\|E(\beta) \phi_{1}\right\|^{2}=\lim _{k \rightarrow \infty}\left\|E\left(\beta_{k}\right) \phi_{1}\right\|^{2} \text {. }
$$

Hence if meas $\beta=0$, then meas $\beta_{k}=0$, and it follows from (9) that $\left\|E\left(\beta_{k}\right) \phi_{1}\right\|^{2}$ $=0$ and from (10) that $\left\|E(\beta) \phi_{1}\right\|^{2}=0$. Thus $\phi_{1} \in H_{a}(C)$. Lemma 1 and the fact that the subspace $H_{a}(C)$ is invariant under $C$ imply that $H_{a}(C)=H$ as was to be shown.

\section{REFERENCES}

1. J. Dombrowski, Spectral properties of phase operators, J. Mathematical Phys. 15 (1974), 576-577.

2. P. R. Halmos, Introduction to Hilbert space and the theory of spectral multiplicity, Chelsea, New York, 1951. MR 13, 563.

3. E. K. Ifantis, Abstract formulation of the quantum mechanical oscillator phase problem, J. Mathematical Phys. 12 (1971), 1021-1026. MR 43 \#5834. $183-186$.

4. E. C. Lerner, Harmonic-oscillator phase operators, Nuovo Cimento 56B (1968),

5. E. C. Lerner, H. W. Huang and G. E. Walters, Some mathematical properties of oscillator phase operators, J. Mathematical Phys. 11 (1970), 1679-1684. MR 41 \#4973.

6. C. R. Putnam, Commutation properties of Hilbert space operators and related topics, Ergebnisse der Mathematik und ihrer Grenzgebiete, Band 36, Springer-Verlag, New York, 1967. MR 36 \#707.

DEPARTMENT OF MATHEMATICS, WRIGHT STATE UNIVERSITY, DAYTON, 